\title{
Valorisation des coques de noyaux de Balanites aegyptiaca (L.) Del. et Hyphaene thébaica (L.) Mart. pour l'élaboration et caractérisation de Charbons Actifs; application pour l'élimination du chrome
}

\author{
Sanda Mamane Ousmaila (Doctorant) \\ Siragi Dounounou Boukari Maâzou (Doctorant) \\ Ecole Doctorale des Sciences Exactes et Techniques (EDSET) \\ Université Abdou Moumouni (UAM), Niger \\ Malam Alma Maman Mousbahou (Maître de Conférences) \\ Natatou Ibrahim (Professeur Titulaire) \\ Département de Chimie de la Faculté des Sciences et Techniques \\ Université Abdou Moumouni (UAM), Niger
}

Doi: 10.19044/esj.2018.v14n21p195 URL:http://dx.doi.org/10.19044/esj.2018.v14n21p195

\begin{abstract}
This paper focuses on the development and characterization of activated carbons to agro-food waste by activation with ortho phosphoric acid, and its application in the elimination of chromium in solution. The functions were determined by Bohem's method. The thermal analysis was carried out on the hulls of waste. The specific surface area and the pore volume are determined according to the method of Brunauer, Emmet and Teller and the method of Barret, Joyner and Halenda respectively. The Lagergren and Ho and McKay models were used for the kinetic study of chromium removal. Those of Langmuir and Freundlich have to do with the study of isotherms and the law of Van't Hoff and Gibbs in relation to the determination of thermodynamic parameters. The results obtained show that the surface functions are acidic in nature. Activated carbon from balanites aegyptiaca has developed a specific surface $\left(1560.7401 \mathrm{~m}^{2} \mathrm{~g}^{-1}\right)$ that exceeds that of Hyphaene thebaica $\left(722.1510 \mathrm{~m}^{2} \mathrm{~g}^{-1}\right.$ ). The pseudo-order 2 kinetics (Ho and McKay) better describe the adsorption with correlation coefficients close to unity. Adsorption capacities and rate constants prove rapid adsorption at the early stages. For the isotherms, the calculated parameter values are much more consistent with those of the Langmuir isotherm. $\Delta \mathrm{G}_{\text {ads }}^{0}<0$; the adsorption process is spontaneous. Moreover, the values are in the range of a physisorption.
\end{abstract}


Keywords: Activated carbon, Balanites aegyptiaca (L.) Del., Chromium, Hyphaene thebaica (L.) Mart, surface area

\section{Résumé}

Ce travail porte sur l'élaboration et caractérisation des charbons actifs avec des déchets agro-alimentaires par activation à l'acide ortho phosphorique, et leur application dans l'élimination du chrome en solution. Les fonctions ont été déterminées par la méthode de Bohem. L'analyse thermique a été effectuée sur les coques de déchets. La surface spécifique et le volume poreux sont déterminés selon la méthode de Brunauer, Emmet et Teller et la méthode de Barret, Joyner et Halenda respectivement. Les modèles de Lagergren et de Ho et McKay ont servi à l'étude cinétique de l'élimination du chrome, ceux de Langmuir et de Freundlich à l'étude des isothermes et la loi de Van't Hoff et la relation de Gibbs à la détermination des paramètres thermodynamiques. Les résultats obtenus montrent que les fonctions de surface sont de nature acide. Le charbon actif issu de balanites aegyptiaca aurait développé un surface spécifique $\left(1560,7401 \mathrm{~m}^{2} \mathrm{~g}^{-1}\right)$ qui dépasse celui de Hyphaene thébaica $\left(722,1510 \mathrm{~m}^{2} \mathrm{~g}^{-1}\right)$. Le cinétique pseudoordre 2 (Ho et McKay) décrit mieux l'adsorption avec des coefficients de corrélation proche de l'unité. Les capacités d'adsorption et les constantes de vitesses prouvent une adsorption rapide aux premières étapes. Pour ce qui est des isothermes, les valeurs des paramètres calculées concordent beaucoup plus à celles de l'isotherme de Langmuir. $\Delta \mathrm{G}_{\text {ads }}^{0}<0$; le processus d'adsorption est spontané. Par ailleurs, les valeurs sont dans la gamme d'une physisorption.

Mots-clés : Balanites aegyptiaca (L.) Del., Charbon actif, Chrome, Hyphaene thebaica (L.) Mart., Surface spécifique

\section{Introduction}

Malgré qu'elle soit indispensable à la vie, l'eau est une ressource très inégalement répartie dans le monde. En outre, elle devient de plus en plus polluée. A ce sujet, les techniques de traitement des cuirs et peaux mises en œuvre dans la tannerie industrielle ou semi-industrielle au Niger utilisant des formulations à base de chrome, tout comme dans plusieurs tanneries industrielles dansle monde (Krishnamoorthy et al., 2012 ; Combéré et al., 2017) ont considérablement contribué à la pollution de l'eau. Le rejet des eaux usées de tannerie génère le plus souvent des effluents ne respectant pas les normes nationales de rejet de chrom9i-ipi, fixées à $1 \mathrm{mg} \mathrm{L}^{-1}$ pour le déversement dans les égouts. Il est donc primordial de limiter le plus possible cette pollution en mettant en place une technique d'élimination du chrome adaptée à notre localité (Niger). Au cours de ces dernières années, l'adsorption demeure l'une des techniques d'élimination de polluants la plus utilisée en 
raison de son efficacité, la facilité de sa mise en œuvre et son coût d'investissement accessible. Cette méthode nécessite le choix d'un adsorbant présentant de bonnes caractéristiques. L'utilisation du charbon actif (CA) comme adsorbant, représente un intérêt dans le traitement des eaux de rejets industriels (Ousmaila et al., 2016). Ceci est justifié par l'importance de la surface spécifique et la porosité développées par ce matériau (Drissa et al, 2009). Ce dernier occupe une place importante dans l'industrie, avec des utilisations diverses (l'extraction de l'or, la filtration de l'air et des polluants organiques, etc.) (SAGEP, 2006), grâce à sa propriété d'adsorber des fluides amenés à son contact. Il est aussi constitué essentiellement de matière carbonée à structure poreuse. Dit autrement, le charbon actif s'agit d'une structure amorphe généralement obtenue après une étape de carbonisation à haute température de biomasses lignocellulosiques et présentant une très grande surface spécifique qui lui confère un fort pouvoir adsorbant (Ousmaila et al., 2016 ; Soleimani \& Kaghazchi, 2008). Il se caractérise également par sa thermostabilité (Chen et al., 2011). Ainsi, de nombreux procédés de fabrication furent mis au point pour permettre une plus grande production de charbon actif à partir de nombreuses matières végétales riches en carbone tels que les graines de palme (Lua et $a l .$, 2006)), le bois de tamarin (Sahu et al., 2010), les coques (Yang et al., 2010), les noix de coco (Gueye et al., 2014), les bagasses de canne à sucre (Cronje et al., 2011), etc., afin de minimiser les coûts de production et de résoudre le problème environnemental lié à leur stockage dans la nature. Le Niger, à l'instar de nombreux autres pays subsahariens, importe du charbon actif utilisé dans divers domaines (traitement de l'Or et le traitement des eaux). Or le pays dispose d'importantes réserves de déchets en matière organique. Pour cette raison, nous avons engagé ce travail qui consiste à élaborer et caractériser du Charbon actif à partir de coques des biomasses lignocellulosiques locales par activation chimique à l'acide ortho-phosphorique $\left(\mathrm{H}_{3} \mathrm{PO}_{4}\right)$ afin d'éliminer le chrome en solution. Les biomasses sélectionnées sont les noyaux de Balanites aegyptiaca (L.) Del. et Hyphaene thebaica (L.) Mart. En effet, ces biomasses proviennent des arbres sauvages très répandus au Niger et qui produisent des fruits saisonniers consommés par la population. Les noyaux de ces fruits se retrouvent dans la décharge municipale comme déchets urbains. Ils constituent alors des déchets agro-alimentaires abondants qui sont plus ou moins difficilement biodégradables. L'utilisation de ces derniers dans ce travail présente un double avantage : élaborer des charbons actifs à faible coût d'une part, et valoriser les déchets afin de leur donner une valeur ajoutée d'autre part. 


\section{Matériel et méthodes}

\section{Matériel végétal}

Il s'agit de coques des noyaux de Balanites aegyptiaca (L.) Del. (figure 1) et de Hyphaene thebaica (L.) Mart. (figure 2).

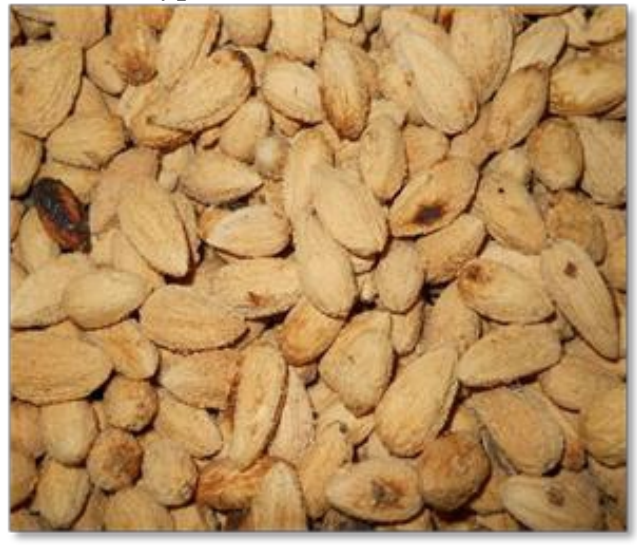

Figure 1. Noyau de Balanites aegyptiaca

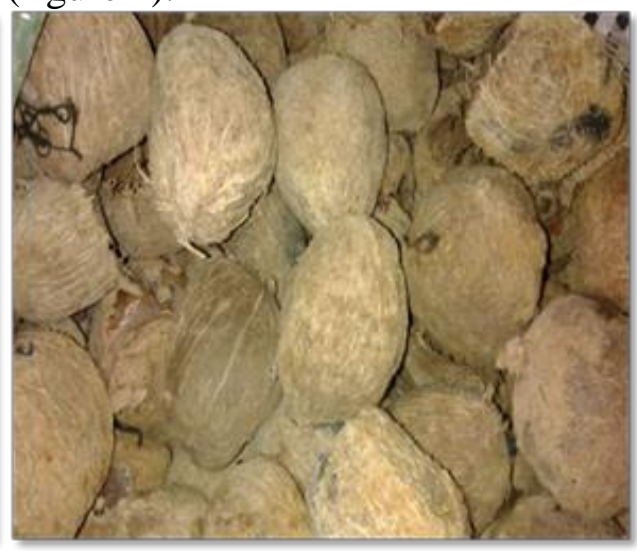

Figure 2. Noyau d' Hyphaene thebaica

\section{Collecte et prétraitement des coques}

Les coques de noyaux des fruits de Balanites aegyptiaca (BA) et de Hyphaene Thebaica (HT) proviennent d'un marché de fruits et légumes de la ville de Niamey (Niger), appelé KATAKO. Les fruits ont été d'abord épluchés et ensuite les noyaux ont été récupérés puis concassés par chute pour séparer les coques du noyau. Ces dernières sont ensuite broyées dans un broyeur à boulets. Après tamisage, les fractions de diamètre compris entre 0.8 et $2 \mathrm{~mm}$ ont été retenues pour l'élaboration des charbons actifs et séchées à l'étuve à $105^{\circ} \mathrm{C}$ pendant $24 \mathrm{~h}$.

\section{La décomposition thermique de coques de BA et HT}

L'analyse thermogravimétrique (ATG) et analyse thermique différentielle (ATD) ont été effectuées sur les coques des noyaux de Balanites aegyptiaca (L.) Del. et de Hyphaene thebaica (L.) Mart. pour voir la décomposition en masse de ces matériaux. Elles sont déterminées à l'aide d'un thermogramme de marque (Lab : METTLER STAR $S W$ 8.10).

\section{Préparation des charbons actifs}

Elle s'effectue en trois étapes (Gueye et al., 2014) :

$\checkmark$ imprégnation de la biomasse dans des solutions de l'agent activant ;

$\checkmark$ carbonisation de la biomasse imprégnée ;

$\checkmark$ purification du produit obtenu. 


\section{Imprégnation}

Dans des béchers de $250 \mathrm{~mL}, 16 \mathrm{~g}$ de coque broyée et $100 \mathrm{~mL}$ de solution d'acide ortho-phosphorique $25 \%$ ont été introduits. Le mélange obtenu est agité pendant $15 \mathrm{~h}$ à la température ambiante. Après filtration sur papier filtre sans cendre, les coques imprégnées sont séchées à l'étuve pendant 24 heures puis refroidies dans un dessiccateur pendant $15 \mathrm{~min}$.

\section{Pyrolyse}

L'échantillon sec obtenu après imprégnation a été placé dans un four à moufle, programmable à haute température (marque TACHETE). La température $\mathrm{du}$ four évolue de façon croissante jusqu'à atteindre la température de pyrolyse $\left(450^{\circ} \mathrm{C}\right)$ avec des vitesses de chauffe pour la pyrolyse $\left(2,5^{\circ} \mathrm{C} \mathrm{min}^{-1}\right)$ et un palier isotherme de $1 \mathrm{~h} 30 \mathrm{~min}$ à la fin du chauffage qui représente le temps de séjour dans le four. Le Charbon actif obtenu est refroidi, lavé abondement à l'eau distillée jusqu'à $\mathrm{pH}=7$ puis séché à l'étuve à $105^{\circ} \mathrm{C}$.

\section{Caractérisation}

\section{Fonction de surface}

La fonction de surface est une caractéristique faisant ressortir les groupements fonctionnels (acides et basiques) du Charbon actif. La méthode de Boehm (Tchakala et al., 2012) a été utilisée pour déterminer les groupements fonctionnels des charbons actifs élaborés (CAEs). Les groupements basiques sont dosés dans leur globalité alors que les groupements acides sont dosés séparément. Le protocole expérimental est le suivant : 0,2 $\mathrm{g}$ de charbon actif a été mis en contact avec $20 \mathrm{~mL}$ des solutions aqueuses de $\mathrm{NaOH}, \mathrm{Na}_{2} \mathrm{CO}_{3}, \mathrm{NaHCO}_{3}, \mathrm{C}_{2} \mathrm{H}_{5} \mathrm{ONa}$ et $\mathrm{HCl}$ à $0,1 \mathrm{M}$. Chaque solution est agitée pendant $24 \mathrm{~h}$. Puis le mélange est filtré. Après filtration, $10 \mathrm{~mL}$ de solution sont dosés. Les solutions basiques sont dosées par l'acide chlorhydrique à $0,1 \mathrm{M}$ et la solution acide est dosée par la soude à $0,1 \mathrm{M}$. Le nombre de moles de la fonction cherchée est donné par la formule (1).

$$
n_{\text {é } q R}=N_{i} V_{i}-N_{f} V_{f}
$$

néqR étant le nombre d'équivalent gramme ayant réagi, $\mathrm{N}_{\mathrm{i}} \mathrm{V}_{\mathrm{i}}$ le nombre d'équivalant gramme avant la réaction et $\mathrm{N}_{\mathrm{f}} \mathrm{V}_{\mathrm{f}}$ le nombre d'équivalant gramme après la réaction.

\section{Surface spécifique et volume poreux}

La surface spécifique est déterminée sur un appareil (MICROMERITICS Gemini VII Surface Area \& Porosity) par adsorption de l'azote liquide à 77 $\mathrm{K}$ selon la méthode classique de Brunauer, Emmet et Teller ou BET [2]. Le volume poreux $(\mathrm{Vp})$ est déterminé par le modèle de Barret, Joyner et Halenda ou BJH (à l'adsorption et à la désorption). 


\section{Application dans le traitement de solution du chrome}

L'élimination du chrome (VI) sur le CA a été effectuée comme suit : dans un bécher de $100 \mathrm{~mL}$, une masse $\mathrm{m}$ de $50 \mathrm{mg}$ du Charbon actif pesée à l'aide d'une balance de précision (à $1 / 10000$ près de marque Precisa) a été introduite dans $50 \mathrm{~mL}$ de la solution du chrome de concentration connue. Le mélange est agité pendant un temps bien défini puis filtré à travers un papier filtre et la concentration résiduelle en chrome est mesurée à l'aide d'un spectrophotomètre à flamme (MP-AES). La capacité d'adsorption du chrome est déterminée par la relation (2) :

$$
q_{e q}=\frac{\left(C_{i}-C_{e q}\right) V}{m_{C A}}
$$

$\mathrm{q}_{\mathrm{eq}}$ étant la capacité d'adsorption du chrome exprimée en $\mathrm{mg} \mathrm{g}^{-1}, \mathrm{C}_{\mathrm{i}}$ la concentration initiale de la solution de chrome en $\mathrm{mg} \mathrm{L}^{-1}, \mathrm{C}_{\mathrm{eq}}$ la concentration finale de la solution de chrome en $\mathrm{mg} \mathrm{L}^{-1}$, $\mathrm{V}$ le volume de la solution de chrome en $\mathrm{mL}$, et $\mathrm{m}_{\mathrm{CA}}$ la masse du charbon actif en $\mathrm{g}$.

\section{Cinétiques d'adsorption du Cr (VI) sur le charbon actif}

Les modèles de pseudo-ordre 1 (Coulibaly, 2014) et de pseudo-ordre 2 [Ho et al., 1998) 18] sont testés et présentés ici. Ces modèles sont décrits par les équations (3) et (4) respectivement.

$$
\begin{aligned}
\frac{d q_{t}}{d t} & =k_{1} \cdot\left(q_{e q}-q_{t}\right) \\
\frac{d q_{t}}{d t} & =k_{2} \cdot\left(q_{e q}-q_{t}\right)^{2}
\end{aligned}
$$

qt étant la quantité de Cr (VI) adsorbée au temps t exprimée en $\mathrm{mg} \mathrm{g}^{-1}$, qeq la quantité de $\mathrm{Cr}$ (VI) adsorbée à l'équilibre en $\mathrm{mg} \mathrm{g}^{-1}, \mathrm{k}_{1}$ la constante de vitesse pseudo-ordre 1 en $\min ^{-1}, \mathrm{k}_{2}$ la constante de vitesse pseudo-ordre 2 en $\mathrm{g} \mathrm{mg}^{-1} \min ^{-1}$, et $\mathrm{t}$ le temps en min.

\section{Les isothermes d'adsorption}

Le modèle de Langmuir et le modèle de Freundlich (Coulibaly, 2014) sont testés et présentés ici. Ces modèles sont décrits par les équations (5) et (6) respectivement :

$$
\begin{gathered}
q_{e q}=\frac{q_{\max } k_{L} C_{e q}}{1+k_{L} C_{e q}} \\
q_{e q}=k_{f} C_{e q}^{1 / n f}
\end{gathered}
$$

$\mathrm{q}_{\mathrm{eq}}$ étant la capacité d'adsorption à l'équilibre exprimée en $\mathrm{mg}^{-1}$, $\mathrm{q}_{\max }$ la capacité maximale d'adsorption à l'équilibre $\mathrm{mg} \mathrm{g}^{-1}, \mathrm{C}_{\mathrm{eq}} \mathrm{la}$ concentration de l'adsorbat à l'équilibre en $\mathrm{mg} \mathrm{L}^{-1}, \mathrm{k}_{\mathrm{L}}$ la constante de 
Langmuir en $\mathrm{Lmg}^{-1}, \mathrm{k}_{\mathrm{f}}$ la constante de Freundlich en $\mathrm{L} \mathrm{g}^{-1}$ et $\mathrm{n}_{\mathrm{f}}$ le coefficient de l'équation de Freundlich.

\section{Aspect thermodynamique de l'adsorption} 2014).

Ce phénomène est caractérisé par les équations (7) et (8) (Coulibaly,

$$
\begin{gathered}
\Delta G_{a d s}^{0}=\Delta H_{a d s}^{0}-T \Delta S_{a d s}^{0} \\
\ln k_{d}=\left(-\frac{\Delta H_{a d s}^{0}}{R}\right) \times \frac{1}{T}+\left(\frac{\Delta S_{a d s}^{0}}{R}\right)
\end{gathered}
$$

$\Delta \mathrm{G}_{\text {ads }}^{0}$ étant l'énergie libre standard d'adsorption exprimée en $\mathrm{kJ}^{\mathrm{mol}^{-}}$ ${ }^{1}, \Delta \mathrm{H}_{\text {ads }}^{0}$ l'enthalpie standard d'adsorption en $\mathrm{kJ} \mathrm{mol}^{-1}$, $\mathrm{T}$ la température en $\mathrm{K}^{-}$

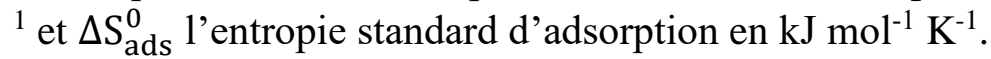

En traçant $\ln \left(\mathrm{k}_{\mathrm{d}}\right)=\mathrm{f}\left(\frac{1}{\mathrm{~T}}\right)$, on obtient une droite de la forme $\mathrm{y}=\mathrm{ax}+\mathrm{b}$ avec $\mathrm{a}=-\frac{\Delta \mathrm{H}_{\mathrm{ads}}^{0}}{\mathrm{R}}$ le coefficient directeur de la droite et $\mathrm{b}=\frac{\Delta \mathrm{S}_{\text {ads }}^{0}}{\mathrm{R}}$ l'ordonné à l'origine. De ces deux relations, on détermine les paramètres thermodynamiques telles que l'enthalpie standard $\left(\Delta \mathrm{H}_{\mathrm{ads}}^{0}=-\mathrm{a} \times \mathrm{R}\right)$ et l'entropie standard $\left(\Delta \mathrm{S}_{\text {ads }}^{0}=\mathrm{b} \times \mathrm{R}\right)$ en valeur absolue. Les enthalpies libres standards sont déterminées en utilisant l'équation (7).

\section{Résultats et discussion}

\section{La décomposition thermique de coques des biomasses utilisées}

La Figure 3 présente les résultats de l'analyse thermique des coques de noyaux de Balanites aegyptiaca (BA) et Hyphaene thebaica (HT) respectivement en rouge et en vert.

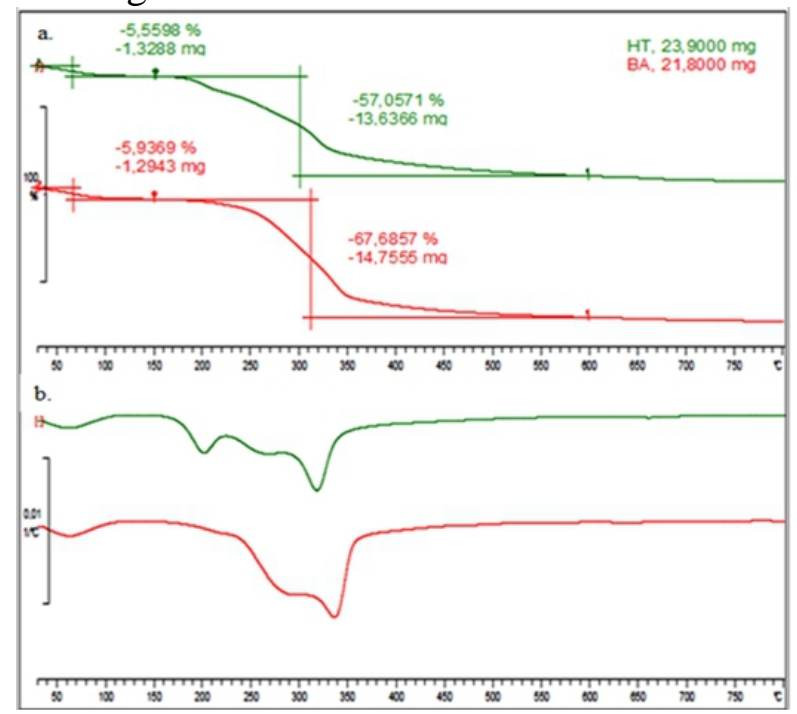

Figure 3. Analyse thermogravimétrique de la coque du noyau de $B A$ et $H T$ 
Pour la partie a. de la figure, on constate que les deux biomasses présentent des thermogrammes qui ont la même allure générale. La perte en masse à faibles températures (entre 50 et $100{ }^{\circ} \mathrm{C}$ ) est due à la déshydratation des biomasses. Elle est légèrement plus importante pour BA que pour HT mais elles sont globalement du même ordre. La seconde perte (de 170 à $600{ }^{\circ} \mathrm{C}$ ), la plus grande perte en masse, est attribuable à l'élimination des matières volatiles et du goudron (Reffas, 2010). La perte (pourcentage) est plus grande pour l'échantillon BA. Ainsi, l'Hyphaene thebaica pourrait avoir un rendement de pyrolyse d'élaboration du charbon actif beaucoup plus important que la Balanites aegyptiaca.

Pour ce qui est des pics observés à la partie b de la Figure 3 obtenus par dérivation de la courbe obtenue dans la partie a, ils sont caractéristiques des différents phénomènes qui se produisent. Le premier (entre 200 et $210{ }^{\circ} \mathrm{C}$ ) qui correspond à la décomposition des hémicelluloses est très marqué chez HT et quasiment absent pour BA. Le deuxième pic (entre 270 et $290^{\circ} \mathrm{C}$ ) est celui de la lignine ; il est plus marqué pour BA que pour HT alors que le troisième, entre 310 et $350^{\circ} \mathrm{C}$, est celui relatif à la cellulose, le constituant majeur des biomasses (Raveendran et al., 1998 ; Yang et al., 2007).

\section{Caractéristiques de Charbons actifs élaborés Fonctions de surface}

Les groupements fonctionnels des charbons actifs sont obtenus grâce à un dosage en retour sélectif (bases de différentes forces et de pka approprié). Ainsi, le $\mathrm{NaHCO}_{3}(\mathrm{pKa}=6,3)$ neutralise seulement les fonctions carboxyliques. Le $\mathrm{Na}_{2} \mathrm{CO}_{3}(\mathrm{pKa}=10,2)$ neutralise les fonctions carboxyliques et les fonctions lactones. Le $\mathrm{NaOH}(\mathrm{pKa}=15,7)$ neutralise les fonctions carboxyliques, lactones et phénoliques. Et le $\mathrm{C}_{2} \mathrm{H}_{5} \mathrm{ONa}(\mathrm{pKa}=20,5)$ neutralise les fonctions carbonyles. Au contraire, les fonctions basiques sont neutralisées dans leur globalité par l'acide chlorhydrique (HCl). Le Tableau I indique les résultats des fonctions de surface des charbons actifs élaborés.

Tableau I. Fonctions de surface en $\mathrm{m}_{\mathrm{eq}} \mathrm{g}^{-1}$

\begin{tabular}{c|ccccc|c}
\hline \multirow{2}{*}{$\begin{array}{c}\text { Charbons } \\
\text { Actifs }\end{array}$} & \multicolumn{5}{|c|}{ Acides } & Basiques \\
\cline { 2 - 7 } & Carboxylique & Lactone & Phénol & Carbonyle & Totaux & Globalité \\
\hline CA-BA & 1,78 & 1,16 & 0,26 & 0,24 & 3,44 & - \\
CA-HT & 1,88 & 0,94 & 0,54 & 0,2 & 3,56 & - \\
& & & & & & \\
\hline
\end{tabular}

L'analyse de ce tableau montre :

L'absence totale de fonction basique. Ceci s'expliquerait par le fait que les charbons actifs élaborés n'ont pas été mis en contact avec de l'oxygène en dessous de $200{ }^{\circ} \mathrm{C}$ ou au-dessus de $700{ }^{\circ} \mathrm{C}$. Les échantillons n'ont pas suivi un traitement avec hydrogène et ils n'ont pas été dégazés à la température ambiante. 
Les fonctions de surface de charbons actifs sont de nature acide. Ces résultats laissent supposer que les échantillons auraient un haut degré d'adsorption. Ces résultats sont similaires aux travaux de Maâzou (Maâzou et al., 2017).

\section{Les isothermes d'adsorption de $\mathbf{N} 2$ sur les charbons actifs élaborés}

Les résultats des isothermes d'adsorption de l'azote $\left(\mathrm{N}_{2}\right)$ sont représentés à la figure 4.

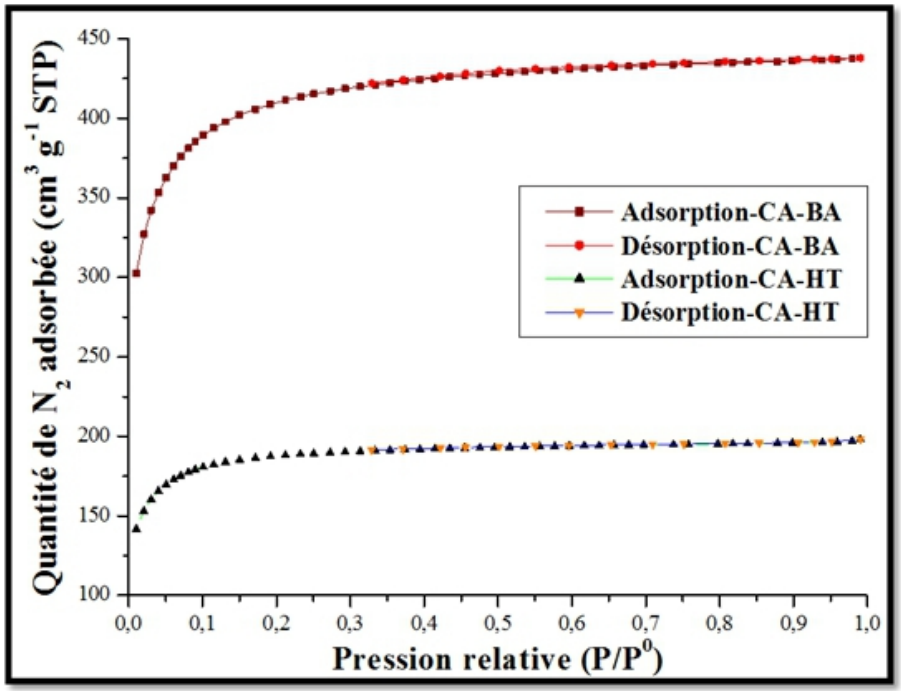

Figure 4. Isothermes d'adsorption de N2

Ces résultats montrent une forte capacité d'adsorption de $\mathrm{N}_{2}$ aux très faibles pressions relatives $\left(\frac{P}{P^{0}} \cong 0,100\right)$ puis un palier traduisant la saturation des charbons actifs, malgré l'augmentation de la pression relative. Les courbes d'adsorption-désorption obtenues ne présentent pas une hystérèse. Les branches de désorption rejoignent les branches d'adsorption pour une pression relative supérieure à 0,32 (figure 4). Ces caractéristiques sont essentiellement des indicatrices de la distribution majoritaire des micropores pour nos échantillons. Les isothermes d'adsorption obtenues seraient essentiellement attribuables à l'isotherme du type I selon la classification BDDT (adopté par l'IUPAC).

\section{Surface spécifique et porosité}

L'équation BET pour l'adsorption de l'azote $\mathrm{N}_{2}$ sur le charbon actif permet de déterminer la surface spécifique de l'échantillon analysé. L'équation BET utilisée en pratique sous sa forme linéaire sur une Gamme de $\frac{\mathrm{P}}{\mathrm{p}^{0}}$ allant de 0,03 à 0,10 est :

$$
\frac{P}{q\left(P^{0}-P\right)}=\frac{1}{V_{m} \times C}+\left(\frac{C-1}{V_{m} \times C}\right) \times \frac{P}{P^{0}}
$$


Le volume de monocouche $\left(\mathrm{V}_{\mathrm{m}}\right)$ et la constante $\mathrm{C}$ sont déterminés à partir de la pente et l'ordonnée à l'origine de droite $\frac{\mathrm{P}}{\mathrm{q}\left(\mathrm{P}^{0}-\mathrm{P}\right)}$ en fonction de $\frac{\mathrm{P}}{\mathrm{P}^{0}}$ . Connaissant $V_{m}$, la surface spécifique $S_{B E T}$ est déterminée à l'aide de l'équation (10).

$$
S_{B E T}=\sigma \cdot \frac{V_{m} \times N}{V_{M}}
$$

La Figure 5 présente les isothermes d'adsorption de $\mathrm{N}_{2}$ sur les charbons actifs élaborés selon la méthode BET.

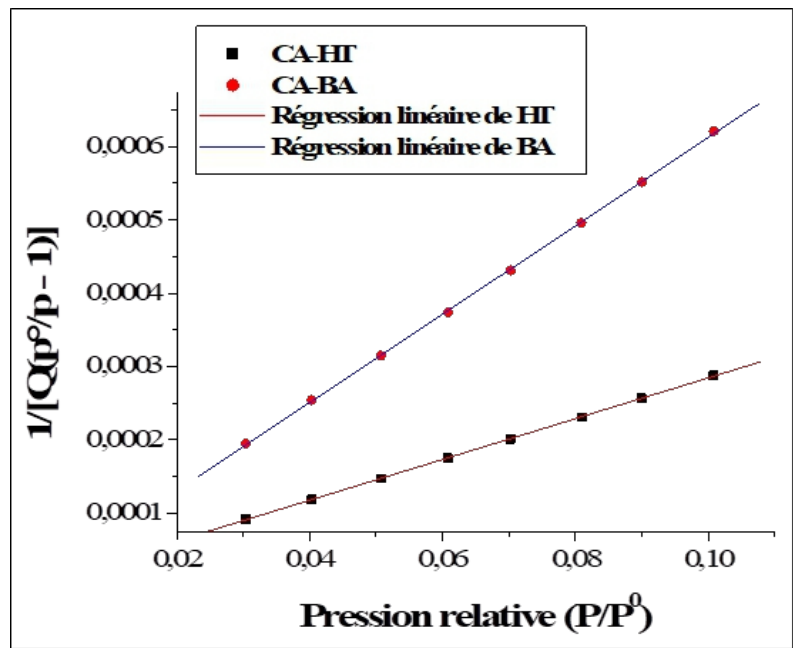

Figure 5. Linéarisation des isothermes BET

Le tableau II indique les surfaces spécifiques et la porosité des charbons actifs élaborés.

Tableau II. Surfaces spécifiques et porosités

Surfaces spécifiques $\left(\mathrm{m}^{2} \mathrm{~g}^{-1} \mathrm{STP}\right)$

\begin{tabular}{|c|c|c|c|c|c|}
\hline & $\mathrm{S}_{\mathrm{BET}}$ & $\mathrm{S}_{\text {micropores }}$ & & $\mathrm{S}_{\text {Externes }}$ & \\
\hline CA-BA & 1560,7401 & 1167,4469 & & 393,2932 & 0,9999535 \\
\hline CA-HT & 722,1510 & 591,7939 & & 130,3571 & 0,9999057 \\
\hline \multicolumn{6}{|c|}{$\begin{array}{l}\text { Volumes poreux } \\
\left(\mathrm{cm}^{3} \mathrm{~g}^{-1} \mathrm{STP}\right)\end{array}$} \\
\hline & $V_{\text {Poreux }}$ & $\mathrm{V}_{\text {Micropores }}$ & $\mathrm{V}_{\text {Externes }}$ & $\frac{V_{\mu}}{V_{T}}(\%)$ & $\frac{V_{E x t}}{V_{T}}(\%)$ \\
\hline CA-BA & 0,677426 & 0,458454 & 0,218972 & 67,675879 & 32,324121 \\
\hline CA-HT & 0,306531 & 0,231967 & 0,074564 & 75,674891 & 24,325109 \\
\hline
\end{tabular}

$(\AA)$ 
Ces résultats montrent que les coefficients de corrélation $\mathrm{R}^{2}$ obtenus sont très significatifs et très proches de l'unité. Ils sont de l'ordre de 0,9999535 et 0,9999057 pour le CA-BA et le CA-HT respectivement. Les surfaces spécifiques $\left(\mathrm{S}_{\mathrm{BET}}\right)$ calculées sont de l'ordre de 1560,7401 et $722,1510 \mathbf{~ m}^{\mathbf{2}} \mathbf{g}^{-1}$ pour le CA-BA et le CA-HT respectivement. Ceci montre que le charbon actif issu de Balanites aegyptiaca aurait développé une surface spécifique qui dépasse celle de charbon actif d'Hyphaene thébaica. Les volumes externes (mésopores et macropores) sont de l'ordre de 0,218972 et $0,074564 \mathrm{~cm}^{3} \mathrm{~g}^{-1}$ pour le CA-BA et le CA-HT respectivement. Les volumes poreux totaux de $\mathrm{N}_{2}$-CAs ( $\mathrm{V}_{\text {poreux }}$ ) sont de 0,306531 et de $0,677426 \mathrm{~cm}^{3} \mathrm{~g}^{-1}$ pour CA-HT et CAHT respectivement. Ainsi, les volumes poreux $\left(\mathrm{V}_{\mathrm{T}}\right)$ sont proportionnels aux surfaces spécifiques. Les volumes externes (meso et macropores) sont déterminés en faisant la différence entre les $\mathrm{V}_{\text {poreux }}$ et les volumes micropores $\left(V_{\mu}\right)$. Les rapports $\frac{V_{\mu}}{V_{T}}$ dépassent $\frac{V_{E x t}}{V_{T}}$ obtenus pour ces échantillons. Ceci indiquerait l'existence de la distribution majoritaire de micropores par rapport à celle des mésopores et macropores, ce qui confirmerait l'attribution essentielle des isothermes d'adsorption au type I (figure 4). La taille d'un pore peut être décrite par son diamètre moyen. Les valeurs des diamètres moyens des pores déterminés par la méthode BET sont inférieures à $20 \AA$ ( $2 \mathrm{~nm})$. Celles-ci seraient attribuables à la distribution de taille des micropores. En outre, ces valeurs dépassent $7 \AA$. Ceci indiquerait qu'ils seraient des supermicropores. Ces échantillons seraient capables d'adsorber des molécules des tailles de micropores, ce qui laisse présager qu'ils seraient des bons adsorbants pour les applications industrielles.

\section{Application de charbons actifs sur l'élimination du chrome Cinétiques d'adsorption}

Les résultats des paramètres caractéristiques des cinétiques d'adsorption de chrome sur les charbons actifs élaborés sont regroupés dans le tableau III.

Tableau III. Paramètres des cinétiques d'adsorption

\begin{tabular}{ccccc}
\hline \multicolumn{5}{c}{ Cinétique de Pseudo-ordre 1 } \\
\hline Paramètres & $\begin{array}{c}\mathrm{q}_{\mathrm{eq}, \text { exp }} \\
\left(\mathrm{mg} \mathrm{g}^{-1}\right)\end{array}$ & $\begin{array}{c}\mathrm{q}_{\mathrm{eq}, \mathrm{cal}} \\
\left(\mathrm{mg} \mathrm{g}^{-1}\right)\end{array}$ & $\begin{array}{c}\mathrm{k}_{1} \\
\left(\mathrm{~min}^{-1}\right)\end{array}$ & $\mathrm{R}^{2}$ \\
\hline CA-BA & 37,54000 & 41,13959 & 0,07058 & 0,97971 \\
CA-HT & 49,13000 & 97,82277 & 0,12189 & 0,96075 \\
\hline \multicolumn{5}{c}{ Cinétique de Pseudo-ordre 2 } \\
\hline Paramètres & $\mathrm{q}_{\mathrm{eq}, \text { exp }}$ & $\mathrm{q}_{\mathrm{eq}, \mathrm{cal}}$ & $\mathrm{k}_{2}$ & $\mathrm{R}^{2}$ \\
& $\left(\mathrm{mg} \mathrm{g}^{-1}\right)$ & $\left(\mathrm{mg} \mathrm{g}^{-1}\right)$ & $\left(\mathrm{g} \mathrm{mg}^{-1} \mathrm{~min}^{-1}\right)$ & \\
\hline CA-BA & 37,54000 & 37,86444 & 0,01613 & 0,99987 \\
CA-HT & 49,13000 & 49,77600 & 0,01508 & 0,99997 \\
\hline
\end{tabular}


Les résultats de ces modélisations montrent que :

$\checkmark \quad$ les coefficients de corrélation $\mathrm{R}^{2}$ obtenus sont de l'ordre de 0,97971 et 0,96075 pour CA-BA et CA-HT (cinétique pseudo-ordre 1) respectivement. Ceci indiquerait que le CA-BA s'adapte mieux à ce modèle que le CA-HT. La capacité d'adsorption de chrome calculée avec le CA-BA $\left(41,13959 \mathrm{mg} \mathrm{g}^{-1}\right)$ est plus faible que celle obtenue avec CA-HT $(97,82277$ $\left.\mathrm{mg} \mathrm{g}^{-1}\right)$. Il en est de même pour les valeurs des constantes de vitesses des CAEs calculées, qui sont de l'ordre de 0,07058 et $0,12189 \mathrm{~min}^{-1}$ pour le CA-BA et CA-HT respectivement.

$\checkmark \quad$ pour la cinétique pseudo-ordre 2, les coefficients de corrélation $\mathrm{R}^{2}$ obtenus sont très significatifs et très proches de l'unité $(1)$. Ils sont de l'ordre de 0,99987 et 0,99997 pour le CA-BA et CA-HT respectivement. Ceci indiquerait que le CA-HT s'adapte mieux à ce modèle que le CA-BA. La capacité d'adsorption de chrome calculée avec le CA-BA (37,86444 $\left.\mathrm{mg} \mathrm{g}^{-1}\right)$ est plus faible que celle obtenue avec le CA-HT $\left(49,77600 \mathrm{mg} \mathrm{g}^{-1}\right)$. Les valeurs des constantes des vitesses des CAEs calculées sont de l'ordre de 0,016613 et $0,01508 \mathrm{~g} \mathrm{mg}^{-1} \mathrm{~min}^{-1}$ pour CA-BA et CA-HT respectivement.

l'application de deux modèles de réaction de surface montre que le modèle pseudo second ordre décrit mieux la totalité des résultats expérimentaux obtenus (coefficients très proches de l'unité et aussi une bonne corrélation entre les valeurs de $\mathrm{q}_{\mathrm{eq} \text { exp }}$ et $\mathrm{q}_{\mathrm{eq}, \mathrm{cal}}$ ). La conformité au modèle pseudo second ordre semble indiquer l'existence d'une chimisorption qui implique des échanges d'électrons à l'interface des Charbons actifs élaborés et le $\mathrm{Cr}$. Cela pourrait révéler une hétérogénéité des charbons actifs élaborés. Des paramètres cinétiques similaires ont été rapportés par Gueye en 2014, pour l'adsorption des $\mathrm{Cr}$ sur les charbons actifs à base de coques d'arachide et le bois de jatropha.

\section{Isothermes d'adsorption du chrome en solution}

Les résultats des paramètres caractéristiques des isothermes d'adsorption de chrome sur les charbons actifs élaborés sont regroupés dans le tableau IV.

Tableau IV. Paramètres des isothermes d'adsorption

\begin{tabular}{ccccc}
\hline \multicolumn{5}{c}{ Isotherme de Langmuir } \\
\hline Paramètres & $\begin{array}{c}\mathrm{q}_{\max } \\
\left(\mathrm{mg} \mathrm{g}^{-1}\right)\end{array}$ & $\begin{array}{c}\mathrm{k}_{\mathrm{L}} \\
\left(\mathrm{L} \mathrm{mg}^{-1}\right)\end{array}$ & $\mathrm{R}_{\mathrm{L}}$ & $\mathrm{R}^{2}$ \\
& 87,64241 & 0,10334 & 0,16215 & 0,90977 \\
CA-BA & 80,90614 & 0,85065 & 0,02297 & 0,98651 \\
CA-HT & \multicolumn{4}{c}{ Isotherme de Freundlich } \\
\hline \multicolumn{7}{c}{$\mathrm{n}_{\mathrm{f}}$} & $1 / \mathrm{n}_{\mathrm{f}}$ & $\begin{array}{c}\mathrm{k}_{\mathrm{f}} \\
\left(\mathrm{min}^{-1}\right)\end{array}$ & $\mathrm{R}^{2}$ \\
\hline Paramètres & & & 9,37606 & 0,94235 \\
& 0,74977 & 1,33373 & 37,02154 & 0,80416 \\
\hline CA-BA & 2,71076 & 0,36890 &
\end{tabular}


L'analyse des résultats de l'isotherme de Langmuir montre que les coefficients de corrélation $\mathrm{R}^{2}$ obtenus sont de l'ordre de 0,90977 et 0,98651 pour le CA-BA et le CA-HT respectivement. Ceci indiquerait que le CA-HT s'adapte mieux à ce modèle que le CA-BA. La capacité maximale d'adsorption de chrome calculée avec le CA-HT $\left(80,90614 \mathrm{mg} \mathrm{g}^{-1}\right)$ est plus faible que celle obtenue avec le CA-BA $\left(87,64241 \mathrm{mg} \mathrm{g}^{-1}\right)$. Les valeurs des constantes de Langmuir des CAEs calculées ne suivent pas la même tendance et sont de l'ordre de 0,10334 et $0,85065 \mathrm{~L} \mathrm{mg}^{-1}$. Les valeurs de relation de Langmuir $\left(\mathrm{R}_{\mathrm{L}}\right)$ sont de l'ordre de 0,16215 et 0,02297 pour le CA-BA et le CAHT respectivement. Ces valeurs sont comprises entre 0 et 1 ; ce qui laisse supposer que le processus d'adsorption de chrome sur les CAEs serait favorable. Pour le modèle de Freundlich, les résultats montrent que les coefficients de corrélation $\mathrm{R}^{2}$ obtenus sont de l'ordre de 0,94235 et 0,80416 pour le CA-BA et le CA-HT respectivement. La valeur de la constante de Freundlich calculée avec le CA-BA $(9,37606)$ est plus faible que celle obtenue avec le CA-HT $(37,02154)$. Le facteur d'hétérogénéité $\left(1 / \mathrm{n}_{\mathrm{f}}\right)$ du CA-HT est inférieur à 1 , ce qui semble indiquer que le couple $\mathrm{Cr} / \mathrm{CA}-\mathrm{HT}$ serait favorable à l'adsorption.

\section{Aspect thermodynamique d'élimination du chrome}

Le tableau $\mathrm{V}$ indique les paramètres thermodynamiques d'élimination du chrome.

Tableau V : Paramètres thermodynamiques

\begin{tabular}{|c|c|c|c|c|c|}
\hline Paramètres & $\begin{array}{c}\text { Température } \\
\left({ }^{\circ} \mathrm{C}\right)\end{array}$ & $\underset{\left(\mathrm{kJ} \mathrm{mol}^{-1}\right)}{\Delta \mathbf{H}_{\mathrm{ads}}^{\mathbf{0}}}$ & $\begin{array}{c}\Delta \mathbf{S}_{\text {ads }}^{\mathbf{0}} \\
\left(\mathrm{kJ} \mathrm{mol}^{-1} \mathrm{~K}^{-1}\right)\end{array}$ & $\underset{\left(\mathrm{kJ} \mathrm{mol}^{-1}\right)}{\Delta \mathbf{G}_{\mathbf{a d i s}}^{\mathbf{0}}}$ & $\mathrm{R}^{2}$ \\
\hline \multirow{5}{*}{ CA-BA } & 40 & & & $-5,73176$ & \\
\hline & 60 & & & $-7,83276$ & \\
\hline & 80 & 27,14889 & 0,10505 & $-9,93376$ & 0,98412 \\
\hline & 100 & & & $-12,03476$ & \\
\hline & 120 & & & $-14,13576$ & \\
\hline \multirow{5}{*}{ CA-HT } & 40 & & & $-6,29095$ & \\
\hline & 60 & & & $-7,51455$ & \\
\hline & 80 & 12,85839 & 0,06118 & $-8,73815$ & 0,97712 \\
\hline & 100 & & & $-9,96175$ & \\
\hline & 120 & & & $-11,18535$ & \\
\hline
\end{tabular}

Les résultats de cette modélisation montrent que les coefficients de corrélation $\mathrm{R}^{2}$ obtenus sont de l'ordre de 0,98412 et 0,97712 pour le CA-BA et le CA-HT respectivement. Les valeurs de l'enthalpie libre standard $\left(\Delta \mathrm{G}_{\mathrm{ads}}^{0}\right)$ diminuent lorsque la température augmente et elles sont négatives tandis que les constantes de distribution augmentent avec la température. L'enthalpie et l'entropie standard $\left(\Delta \mathrm{H}_{\mathrm{ads}}^{0}\right.$ et $\left.\Delta \mathrm{S}_{\mathrm{ads}}^{0}\right)$ sont de l'ordre de $27,14889 \mathrm{~kJ} \mathrm{~mol}^{-1}$ et de $0,10505 \mathrm{~kJ} \mathrm{~mol}^{-1} \mathrm{~K}^{-1}$ pour le CA-BA, et elles sont de l'ordre de $12,85839 \mathrm{~kJ}$ 
$\mathrm{mol}^{-1}$ et $0,06118 \mathrm{~kJ} \mathrm{~mol}^{-1} \mathrm{~K}^{-1}$ pour le CA-HT. Ceci indiquerait que l'adsorption de chrome sur les CAEs serait spontanée. Par ailleurs, les valeurs de l'enthalpie d'adsorption obtenues sont comprises entre 5 et $40 \mathrm{~kJ} \mathrm{~mol}^{-1}$ et celles de l'enthalpie libre $\Delta \mathrm{G}_{\mathrm{ads}}^{0}$ sont comprises entre -20 et $0 \mathrm{~kJ} \mathrm{~mol}^{-1}$. Cellesci peuvent supposer que les mécanismes de sorption entre le $\mathrm{Cr}$ et les charbons actifs élaborés seraient dominés par un processus de nature physique (Coulibaly, 2014 ; HUGUET, 2009).

\section{Conclusion}

Les charbons actifs ont été élaborés à partir des coques de noyaux de Balanites aegyptiaca (BA) et Hyphaene thebaica (HT) par activation chimique à l'acide $\mathrm{H}_{3} \mathrm{PO}_{4}$. Ces charbons ont été caractérisés et utilisés pour l'adsorption de Chrome. Il ressort de cette étude que :

$\checkmark$ les trois pics observés sur les thermogrammes de coques de BA et HT sont caractéristiques des hémicelluloses, de la lignine et de la cellulose ;

$\checkmark$ les fonctions de surface des charbons actifs sont de nature acide ;

$\checkmark$ les isothermes d'adsorption obtenues seraient essentiellement attribuables à l'isotherme du type I ;

$\checkmark \quad$ les valeurs des diamètres moyens des pores déterminés sont inférieures à $20 \AA(2 \mathrm{~nm})$;

$\checkmark$ les modèles pseudo-ordre 2 et de Langmuir décrivent une meilleure adsorption du chrome sur le charbon actif ;

$\checkmark$ il y aurait présence d'une chimisorption au sein de l'adsorption ;

$\checkmark$ des échanges d'électrons à l'interface de charbons actifs/ $\mathrm{Cr}$ et une hétérogénéité des charbons actifs sont constatés ;

$\checkmark$ l'adsorption de chrome sur les charbons actifs serait de nature physique.

Les charbons actifs élaborés seraient capables d'adsorber des molécules des tailles de micropores.

\section{References:}

1. Chenn, $Y$ et al. (2011). Application studies of carbon derived from rice husks produced by chemical- thermal process. A review Adv. Colloïd interface Sci 163, 39-52.

2. Combéré, W., Arsène, H. Y., Abdoulaye, D., \& Kaboré, L. (2017). Elimination du chrome trivalent des eaux par des zéolithes échangées au fer et des argiles naturelles du burkina faso: J. Soc. Ouest-Afr. Chim. 043, 26- 30.

3. Coulibaly, S. L. (2014). Abattement des phosphates des eaux usées par adsorption sur des géomatériaux constitués de Latérite, grès et 
schistes. Thèse de doctorat en Co-Tutelle à l'Université de Lorraine (LL), Nancy-France NF et de L'Université Nangui Abrogoua (UNA), Abidjan/ Cote d'Ivoire ACI, 213.

4. Cronje, K.J., Chetty, K., Carsky, M., Sahu, J.N., \& Meikap, B.C. (2011). Optimization of chromium (VI) sorption potential using developed activated carbon from sugarcane bagasse with chemical activation by zinc chloride. Journal Desalination 275, 276-284.

5. Drissa, B., Bini, D., Albert, T., Guessan, E. Z., Grah, P. A., Didier, R., \& Jean, V. W. (2009). Etudes comparées des méthodes de préparation du charbon actif, suivie d'un test de dépollution d'une eau contaminée au Diuron : J. Soc. Ouest-Afr. Chim. 028, 41-52.

6. Gueye, M., Richardson, Y., Kafack, F.T., \& Blin, J. (2014). High efficiency activated carbons from african biomass residues for the removal of chromium (VI) from wastewater : Journal of environmental chemical engineering 2 (1), 273-281. DOI : http://doi.org/101007/s10450-017-9929-7.

7. Ho, Y. S., \& McKay, G. (1998). Sorption of dye from aqueous solution by peat. Chem. Eng. J., 70 (2), $115-124$.

8. Huguet, S. (2009). Etude du devenir du cadmium dans un sediment de curage fortement contaminé et des mécanismes d'accumulation du cadmium chez arabidopsis halleri. Thèse de doctorat à l'Université des Sciences et technologies de Lille 1, Lille-France LF, 235.

9. Krishnamoorthy, G., Sadulla, S., \& Sehgal1, P.K. (2012). Asit Baran Mandal, Green chemistry approaches to leather tanning process for making chrome-free leather by unnatural amino acids : Journal of Hazardous Materials, Vol. 215-216, 173-182.

10. Lua, A.C., Lau, F.Y., \& Guo J. (2006). Influence of pyrolysis conditions on pore development of oil-palm-shell activated carbons : Journal of Analytical and Applied Pyrolysis 76, 96-102.

11. Ousmaila, S.M., Adamou, Z., Ibrahim, D., \& Ibrahim, N. (2016). Préparation et caractérisation de charbons actifs à base de coques de noyaux de Balanites eagyptiaca et de Zizyphus mauritiana: J. Soc. Ouest-Afr. Chim. 041, 59- 67.

12. Maâzou, S.D.B., Hima, I. H., Maman Mousbahou, M. A., Adamou, Z., \& Ibrahim, N. (2017). Elimination du chrome par du charbon actif élaboré et caractérisé à partir de la coque du noyau de Balanites agyptiaca : Int. J. Biol. Chem. Sci. 11 (6) : 3050-3065. DOI : https://dx.doi.org/10.4314/ijbcs.v11i6.39.

13. Raveendran, K. \& Ganesh, A. (1998). Adsorption characteristics and pore-development of biomass-pyrolysis char. Fuel. 77 (7), 769-781.

14. Reffas, A. (2010). Etude de l'adsorption de colorants organiques (rouge nylosan et bleu de méthylène) sur des charbons actifs préparés 
à partir de marc de café. Thèse de doctorat en génie de l'environnement à l'Université Mentouri-Contatine, Algérie AE, 153.

15. Sahu, J.N., Acharya, J., \& Meikap, B.C. (2010). Optimization of production conditions for activated carbons from Tamarind wood by zinc chloride using response surface methodology: Bioresource Technology 101, 1974-1982.

16. Société Anonyme de Gestion des Eaux de Paris (2006). Traitement de potabilisation des eaux de surface, adsorption sur charbon actif : Conf, 2006.

17. Soleimani, M. \& Kaghazchi, T. (2008). Adsorption of gold ions from industrial wastewater using activated carbon derived from hard shell of apricot stones : An agricultural waste, Bioresourtechno 99, 53745383.

18. Tchakala, I., Bawa, L. M., Djaneye-Boundjou, G., Doni, K.S., \& Nambo, P. (2012). Optimisation du procédé de préparation des charbons actifs par voie chimique $\left(\mathrm{H}_{3} \mathrm{PO}_{4}\right)$ à partir des tourteaux de Karité et des tourteaux de Coton : Int. J. Biol. Chem. Sci. 6 (1), 461478. DOI : http://dx.doi.org/10.4314/ijbcs.v6i1.42

19. Yang, H., Yan, R., Chin, T., Tee, L. D., Chen, H., \& Zheng, C. (2007). Characteristics of hemicellulose, cellulose and lignin pyrolysis : Energy \& Fuels 18, 1781-1788.

20. Yonli, A. H., Kabore, H. A., \& Koulidiati J. (2014). $\alpha$-Endosulfan removal from water by adsorption over natural clays from Burkina Faso : An Isothermal Study, Journal of Materials Science and Chemical Engineering 2, 49-57.

Isotherm Tabular Report CA-BA

\begin{tabular}{cccrc}
\hline $\begin{array}{c}\text { Pression Relative } \\
\left(\mathrm{P} / \mathrm{P}^{0}\right)\end{array}$ & $\begin{array}{c}\text { Pression Absolue } \\
(\mathrm{mmHg})\end{array}$ & $\begin{array}{c}\text { Quantité } \\
\text { Adsorbée } \\
\left(\mathrm{cm}^{3} / \mathrm{g} \mathrm{STP}\right)\end{array}$ & $\begin{array}{c}\text { temps de } \\
\text { contact } \\
(\mathrm{h}: \mathrm{min})\end{array}$ & $\begin{array}{c}\text { Pression de } \\
\text { saturation } \\
(\mathrm{mmHg})\end{array}$ \\
0.010216674 & 8.048897 & 302.6002 & $01: 05$ & 787.169556 \\
0.020331208 & 16.011513 & 326.9923 & $01: 12$ & 787.533752 \\
0.030388908 & 23.931738 & 342.2896 & $01: 17$ & 787.515564 \\
0.040299413 & 31.732044 & 353.4900 & $01: 22$ & 787.407104 \\
0.050764109 & 39.952751 & 362.7118 & $01: 25$ & 787.027527 \\
0.060797017 & 47.877106 & 369.9891 & $01: 29$ & 787.491028 \\
0.070251958 & 55.305653 & 375.7394 & $01: 32$ & 787.247131 \\
0.080940207 & 63.708553 & 381.2612 & $01: 35$ & 787.106384 \\
0.090001430 & 70.849869 & 385.2147 & $01: 38$ & 787.208252 \\
0.100805894 & 79.365433 & 389.2764 & $01: 40$ & 787.309448
\end{tabular}




\begin{tabular}{|c|c|c|c|c|}
\hline 0.114894043 & 90.448547 & 393.8751 & $01: 43$ & 787.234436 \\
\hline 0.130980731 & 103.072594 & 397.7674 & 01:44 & 786.929443 \\
\hline 0.149891072 & 117.962700 & 402.3182 & 01:47 & 786.989502 \\
\hline 0.171418373 & 134.919296 & 405.7578 & 01:48 & 787.076050 \\
\hline 0.191730709 & 150.920563 & 408.8027 & 01:50 & 787.148621 \\
\hline 0.212088278 & 166.954575 & 411.3712 & 01:51 & 787.193787 \\
\hline 0.232432775 & 182.926453 & 413.5685 & 01:53 & 787.007996 \\
\hline 0.250400896 & 197.066040 & 415.3028 & $01: 54$ & 787.002136 \\
\hline 0.272706343 & 214.597855 & 417.1320 & $01: 56$ & 786.919189 \\
\hline 0.292871181 & 230.497574 & 418.6277 & 01:58 & 787.027161 \\
\hline 0.313052115 & 246.436310 & 420.0247 & 01:59 & 787.205383 \\
\hline 0.333673418 & 262.513733 & 421.1983 & 02:01 & 786.738525 \\
\hline 0.353498550 & 278.183746 & 422.2814 & 02:02 & 786.944519 \\
\hline 0.373883588 & 294.272034 & 423.3303 & 02:04 & 787.068604 \\
\hline 0.394316851 & 310.308685 & 424.2462 & 02:05 & 786.952637 \\
\hline 0.414445494 & 326.143738 & 425.1131 & 02:07 & 786.940002 \\
\hline 0.434826691 & 342.185516 & 425.9281 & 02:08 & 786.946899 \\
\hline 0.455020029 & 358.037720 & 426.6843 & 02:10 & 786.861450 \\
\hline 0.475217564 & 374.007477 & 427.4141 & 02:11 & 787.023682 \\
\hline 0.495508109 & 389.965698 & 428.0628 & $02: 13$ & 787.001648 \\
\hline 0.515905218 & 405.868561 & 428.7057 & 02:14 & 786.711487 \\
\hline 0.535696245 & 421.643494 & 429.2900 & $02: 16$ & 787.094360 \\
\hline 0.556469404 & 437.934357 & 429.8547 & $02: 17$ & 786.987305 \\
\hline 0.576636644 & 453.669373 & 430.4044 & 02:19 & 786.750854 \\
\hline 0.596540994 & 469.381531 & 430.9125 & 02:20 & 786.838684 \\
\hline 0.616762593 & 485.347778 & 431.3608 & $02: 22$ & 786.928040 \\
\hline 0.636996238 & 501.202240 & 431.8551 & $02: 23$ & 786.821350 \\
\hline 0.656975314 & 516.876892 & 432.2842 & $02: 25$ & 786.752380 \\
\hline 0.676984934 & 532.655457 & 432.6441 & $02: 26$ & 786.805481 \\
\hline 0.697105689 & 548.490662 & 433.0756 & $02: 28$ & 786.811340 \\
\hline 0.737145630 & 580.130371 & 433.7078 & $02: 29$ & 786.995605 \\
\hline 0.757654609 & 596.117920 & 434.1233 & $02: 31$ & 786.793762 \\
\hline 0.797736599 & 627.595703 & 434.7005 & $02: 32$ & 786.720459 \\
\hline 0.817527952 & 643.429077 & 435.0585 & $02: 34$ & 787.042297 \\
\hline 0.838090208 & 659.423279 & 435.3059 & $02: 36$ & 786.816589 \\
\hline 0.878055228 & 690.875977 & 435.8145 & $02: 37$ & 786.825195 \\
\hline 0.898202708 & 706.722107 & 436.1350 & 02:39 & 786.818054 \\
\hline 0.938616690 & 738.600647 & 436.5815 & $02: 40$ & 786.903381 \\
\hline
\end{tabular}




\begin{tabular}{lllll}
\hline 0.958958050 & 754.639465 & 436.9880 & $02: 42$ & 786.936890 \\
0.979276633 & 770.626099 & 437.4418 & $02: 43$ & 786.934021 \\
0.990476643 & 779.453369 & 437.9531 & $02: 45$ & 786.947754 \\
0.951689567 & 748.815247 & 437.2952 & $02: 49$ & 786.827209 \\
0.928520542 & 730.587891 & 437.0346 & $02: 50$ & 786.830078 \\
0.905925555 & 712.736267 & 436.7812 & $02: 52$ & 786.749268 \\
0.853613091 & 671.585205 & 436.2023 & $02: 53$ & 786.755981 \\
0.807497357 & 635.384155 & 435.6447 & $02: 55$ & 786.856018 \\
0.751554145 & 591.341187 & 434.9021 & $02: 56$ & 786.824463 \\
0.709310134 & 558.081482 & 434.2568 & $02: 58$ & 786.794739 \\
0.653395604 & 514.033508 & 433.3782 & $02: 59$ & 786.710999 \\
0.595765107 & 468.617401 & 432.2556 & $03: 01$ & 786.580811 \\
0.550312213 & 433.070587 & 431.2620 & $03: 03$ & 786.954346 \\
0.501576273 & 394.657288 & 430.1093 & $03: 05$ & 786.834045 \\
0.456109763 & 358.839874 & 428.1962 & $03: 06$ & 786.740173 \\
0.421703046 & 331.737396 & 426.4263 & $03: 08$ & 786.661133 \\
0.371399920 & 292.159149 & 424.1042 & $03: 09$ & 786.643005 \\
0.329036545 & 258.802887 & 421.9300 & $03: 11$ & 786.547546 \\
\hline
\end{tabular}

Isotherm Tabular Report CA-HT

\begin{tabular}{ccccc}
\hline $\begin{array}{c}\text { Pression } \\
\text { Relative } \\
(\mathrm{P} / \mathrm{P} 0)\end{array}$ & $\begin{array}{c}\text { Pression } \\
\text { Absolue } \\
(\mathrm{mmHg})\end{array}$ & $\begin{array}{c}\text { Quantité } \\
\text { Adsorbée }\left(\mathrm{cm}^{3} / \mathrm{g}\right. \\
\text { STP })\end{array}$ & $\begin{array}{c}\text { temps de } \\
\text { contact } \\
(\mathrm{h}: \mathrm{min})\end{array}$ & $\begin{array}{c}\text { Pression de } \\
\text { saturation } \\
(\mathrm{mmHg})\end{array}$ \\
0.010231129 & 8.090036 & 141.7646 & $01: 01$ & 789.163147 \\
0.020312080 & 16.063156 & 153.0548 & $01: 06$ & 790.727600 \\
0.030361778 & 24.009516 & 160.3061 & $01: 10$ & 790.780945 \\
0.040294564 & 31.861967 & 165.5585 & $01: 14$ & 790.726196 \\
0.050736713 & 40.121189 & 169.7130 & $01: 18$ & 790.772339 \\
0.060825776 & 48.098061 & 172.9468 & $01: 21$ & 790.751282 \\
0.070272535 & 55.565746 & 175.3685 & $01: 24$ & 790.717834 \\
0.080907292 & 63.975273 & 177.6245 & $01: 26$ & 790.723206 \\
0.090043854 & 71.196869 & 179.2371 & $01: 29$ & 790.691040 \\
0.100839780 & 79.733566 & 180.7299 & $01: 31$ & 790.695557 \\
0.114971235 & 90.885582 & 182.3607 & $01: 32$ & 790.507141 \\
0.130938861 & 103.540512 & 183.8941 & $01: 34$ & 790.754639 \\
0.149931180 & 118.537537 & 185.2598 & $01: 35$ & 790.612976 \\
0.171469271 & 135.558777 & 186.4980 & $01: 37$ & 790.571838 \\
0.191846302 & 151.648331 & 187.4152 & $01: 39$ & 790.467834 \\
\hline
\end{tabular}




\begin{tabular}{|c|c|c|c|c|}
\hline 0.212115806 & 167.676224 & 188.1723 & 01:40 & 790.493774 \\
\hline 0.232403907 & 183.715118 & 188.7959 & 01:42 & 790.499268 \\
\hline 0.250383487 & 197.948105 & 189.3025 & 01:44 & 790.579712 \\
\hline 0.272714742 & 215.585464 & 189.8185 & $01: 45$ & 790.516357 \\
\hline 0.292895134 & 231.544830 & 190.2616 & 01:47 & 790.538330 \\
\hline 0.313246264 & 247.578842 & 190.6484 & $01: 48$ & 790.364868 \\
\hline 0.333518416 & 263.615875 & 191.0125 & 01:50 & 790.408752 \\
\hline 0.353663715 & 279.532593 & 191.3348 & 01:51 & 790.390930 \\
\hline 0.373902644 & 295.565613 & 191.6538 & 01:53 & 790.488159 \\
\hline 0.394224712 & 311.641541 & 191.9330 & $01: 54$ & 790.517517 \\
\hline 0.414482352 & 327.646149 & 192.2078 & $01: 56$ & 790.494812 \\
\hline 0.434804913 & 343.729950 & 192.4641 & 01:58 & 790.538330 \\
\hline 0.455024882 & 359.667175 & 192.7062 & 01:59 & 790.434082 \\
\hline 0.475314232 & 375.697693 & 192.9304 & 02:01 & 790.419617 \\
\hline 0.495440544 & 391.615692 & 193.1262 & 02:02 & 790.439331 \\
\hline 0.515861635 & 407.680847 & 193.3143 & 02:04 & 790.291077 \\
\hline 0.536064963 & 423.613068 & 193.5146 & 02:05 & 790.227112 \\
\hline 0.556299359 & 439.695496 & 193.6868 & 02:07 & 790.393677 \\
\hline 0.576537701 & 455.645233 & 193.8651 & 02:08 & 790.312988 \\
\hline 0.596603215 & 471.517944 & 194.0239 & $02: 10$ & 790.337585 \\
\hline 0.616822725 & 487.491180 & 194.1861 & 02:11 & 790.326233 \\
\hline 0.636814359 & 503.283630 & 194.3290 & $02: 13$ & 790.314514 \\
\hline 0.656923138 & 519.159973 & 194.4692 & $02: 14$ & 790.290283 \\
\hline 0.677056534 & 535.062439 & 194.5955 & $02: 16$ & 790.277344 \\
\hline 0.697080206 & 550.906860 & 194.7275 & $02: 17$ & 790.306274 \\
\hline 0.737352993 & 582.678711 & 194.9139 & $02: 19$ & 790.230347 \\
\hline 0.757506950 & 598.555786 & 195.0818 & $02: 20$ & 790.165405 \\
\hline 0.797581401 & 630.282471 & 195.2853 & $02: 22$ & 790.242188 \\
\hline 0.817768775 & 646.280457 & 195.4109 & $02: 23$ & 790.297302 \\
\hline 0.837859792 & 662.104126 & 195.5328 & $02: 25$ & 790.232605 \\
\hline 0.878247081 & 693.897583 & 195.7651 & $02: 26$ & 790.093811 \\
\hline 0.898263949 & 709.816956 & 195.9166 & $02: 28$ & 790.209778 \\
\hline 0.938867626 & 741.841980 & 196.2695 & $02: 29$ & 790.145447 \\
\hline 0.958922531 & 757.753296 & 196.5991 & $02: 31$ & 790.213257 \\
\hline 0.979330136 & 773.834229 & 197.1980 & $02: 33$ & 790.166870 \\
\hline 0.990425474 & 782.660034 & 198.1708 & $02: 35$ & 790.226074 \\
\hline 0.951746494 & 751.916443 & 196.6786 & $02: 38$ & 790.038574 \\
\hline 0.928367364 & 733.509094 & 196.3386 & $02: 40$ & 790.106506 \\
\hline
\end{tabular}




\begin{tabular}{lllll}
\hline 0.906022983 & 715.707336 & 196.1378 & $02: 42$ & 789.943909 \\
0.853383164 & 674.233276 & 195.8074 & $02: 43$ & 790.070984 \\
0.807434054 & 637.965454 & 195.5442 & $02: 45$ & 790.114624 \\
0.751454635 & 593.724426 & 195.2901 & $02: 46$ & 790.100159 \\
0.709177012 & 560.290527 & 195.0607 & $02: 48$ & 790.057373 \\
0.653120967 & 516.060120 & 194.7380 & $02: 49$ & 790.144775 \\
0.595687404 & 470.676636 & 194.3586 & $02: 51$ & 790.140320 \\
0.550642580 & 434.999908 & 194.0158 & $02: 53$ & 789.985962 \\
0.501390770 & 396.104553 & 193.6323 & $02: 55$ & 790.011658 \\
0.455864330 & 360.173248 & 193.1238 & $02: 56$ & 790.088684 \\
0.421582898 & 333.065491 & 192.7508 & $02: 58$ & 790.035583 \\
0.371216882 & 293.272552 & 192.1313 & $03: 00$ & 790.030212 \\
0.328831848 & 259.826874 & 191.4898 & $03: 01$ & 790.151184 \\
\hline
\end{tabular}

BET Surface Area Report CA-BA

BET Surface Area: $1560.7401 \pm 6.1472 \mathrm{~m}^{2} / \mathrm{g}$

Slope: $0.002783 \pm 0.000011 \mathrm{~g} / \mathrm{cm}^{3} \mathrm{STP}$

Y-Intercept: $0.000006 \pm 0.000001 \mathrm{~g} / \mathrm{cm}^{3} \mathrm{STP}$

$$
\text { C: } 440.353856
$$

Qm: $358.5272 \mathrm{~cm}^{3} / \mathrm{g}$ STP

Correlation Coefficient: 0.9999535

Molecular Cross-Sectional Area: $0.1620 \mathrm{~nm}^{2}$

$\begin{array}{ccc}\begin{array}{c}\text { Relative } \\ \text { Pressure } \\ \left(\mathrm{p} / \mathrm{p}^{\circ}\right)\end{array} & \text { Quantity } & \begin{array}{c}1 /\left[\mathrm{Q}\left(\mathrm{p}^{\circ} / \mathrm{p}\right.\right. \\ -1)]\end{array} \\ 0.030213506 & 311.1387 & 0.000100 \\ 0.040168571 & 325.0774 & 0.000129 \\ 0.050600996 & 337.5462 & 0.000158 \\ 0.060647860 & 347.9795 & 0.000186 \\ 0.070144690 & 356.9530 & 0.000211 \\ 0.080753569 & 366.1908 & 0.000240 \\ 0.089888294 & 373.5663 & 0.000264 \\ 0.100715366 & 381.6606 & 0.000293\end{array}$

BET Surface Area Report : CA-HT

BET Surface Area: $722.1510 \pm 4.0517 \mathrm{~m}^{2} / \mathrm{g}$

Slope: $0.006018 \pm 0.000034 \mathrm{~g} / \mathrm{cm}^{3} \mathrm{STP}$

Y-Intercept: $0.000010 \pm 0.000002 \mathrm{~g} / \mathrm{cm}^{3} \mathrm{STP}$

$$
\text { C: } 584.233007
$$

Qm: $165.8897 \mathrm{~cm}^{3} / \mathrm{g} \mathrm{STP}$

Correlation Coefficient: 0.9999057

Molecular Cross-Sectional Area: $0.1620 \mathrm{~nm}^{2}$ 


$\begin{array}{ccc}\begin{array}{c}\text { Relative } \\ \text { Pressure } \\ \left(\mathrm{p} / \mathrm{p}^{\circ}\right)\end{array} & \text { Quantity } & \begin{array}{c}1 /\left[\mathrm{Q}\left(\mathrm{p}^{\circ} / \mathrm{p}\right.\right. \\ -1)]\end{array} \\ 0.030054243 & 251.0777 & 0.000123 \\ 0.040032118 & 259.3163 & 0.000161 \\ 0.050417710 & 266.0310 & 0.000200 \\ 0.060543317 & 271.4300 & 0.000237 \\ 0.069958237 & 275.6608 & 0.000273 \\ 0.080679675 & 279.7571 & 0.000314 \\ 0.089757043 & 282.7913 & 0.000349 \\ 0.100717811 & 285.9887 & 0.000392\end{array}$

Kinetics repport : CA-BA

\begin{tabular}{rr} 
Temps en min & \multicolumn{1}{c}{$\mathrm{t}^{1 / 2}$} \\
5 & 2,236068 \\
10 & 3,1622777 \\
15 & 3,8729833 \\
20 & 4,472136 \\
30 & 5,4772256 \\
40 & 6,3245553 \\
50 & 7,0710678 \\
60 & 7,7459667 \\
70 & 8,3666003 \\
80 & 8,9442719 \\
90 & 9,486833 \\
100 & 10 \\
110 & 10,488088 \\
120 & 10,954451
\end{tabular}

$\mathrm{Qt}$

33,97

34,54

34,75

35,03

35,34

35,47

35,78

36,75

36,94

37,13

37,21

37,29

37,42

37,54

Kinetics CA-HT

Temps en min \#VALEUR! \#VALEUR!

$\begin{array}{rr}5 & 2,236068 \\ 10 & 3,1622777 \\ 15 & 3,8729833 \\ 20 & 4,472136 \\ 30 & 5,4772256 \\ 40 & 6,3245553 \\ 50 & 7,0710678\end{array}$

39,79

43,32

45,26

45,32

47,7

48,5

48,87
Qe

37,54

37,54

37,54

37,54

37,54

37,54

37,54

37,54

37,54

37,54

37,54

37,54

37,54

37,54
LNQ

1,2725656

1,0986123

1,0260416

0,9202828

0,7884574

0,7275486

0,5653138

$-0,235722$

$-0,510826$

$-0,891598$

$-1,108663$

$-1,386294$

$-2,120264$

\#\#\#\#\#\#\#\# $\mathrm{t} / \mathrm{Qt}$

0,1471887

0,2895194

0,43165468

0,57093919

0,84889643

1,12771356

1,39742873

1,63265306

1,89496481

2,15459197

2,41870465

2,6816841

2,93960449

3,1965903

\#VALEUR!

49,13 2,2343063

0,12565971

$49,13 \quad 1,7595806$

0,23084026

$49,13 \quad 1,3532545$

0,33141847

$49,13 \quad 1,3376292$

0,44130627

$49,13 \quad 0,3576744$

0,62893082

$49,13 \quad-0,462035$

0,82474227

$49,13 \quad-1,347074$

1,02312257 


\begin{tabular}{|c|c|c|c|c|}
\hline 7,7459667 & 49,03 & 49,13 & $-2,302585$ & 1,22374057 \\
\hline 8,3666003 & 49,03 & 49,13 & $-2,302585$ & 1,42769733 \\
\hline 8,9442719 & 49,03 & 49,13 & $-2,302585$ & 1,63165409 \\
\hline 9,486833 & 49,04 & 49,13 & $-2,407946$ & 1,83523654 \\
\hline 100 & 49,05 & 49,13 & $-2,525729$ & 2,03873598 \\
\hline 10,488088 & 49,1 & 49,13 & $-3,506558$ & 2,24032587 \\
\hline 10,954451 & 49,13 & 49,13 & \#\#\#\#\#\#\#\# & 2,44249949 \\
\hline
\end{tabular}

\begin{tabular}{|r|r|r|c|c|c|c|c|c|}
\hline \multicolumn{1}{r|}{$\mathrm{Ci}$} & \multicolumn{1}{c|}{$\mathrm{Ce}$} & \multicolumn{1}{c|}{$\mathrm{Qe}$} & $1 / \mathrm{Qe}$ & $1 / \mathrm{Ce}$ & Ce/Qe & $\ln Q \mathrm{l}$ & $\ln \mathrm{le}$ & $\ln (\mathrm{Qe} / \mathrm{Ce})$ \\
\hline 0 & 0 & 0 & \#DIV/0! & \#DIV/0! & \#DIV/0! & \#NOMBRE! & \#NOMBRE! & \#DIV/0! \\
\hline 40 & 2,34 & 23,5375 & 0,0424854 & 0,4273504 & 0,0994158 & 3,15859489 & 0,85015093 & 2,30844396 \\
\hline 60 & 2,59 & 35,88125 & 0,0278697 & 0,3861004 & 0,0721825 & 3,58021487 & 0,95165788 & 2,628557 \\
\hline 70 & 2,76 & 42,025 & 0,0237954 & 0,3623188 & 0,0656752 & 3,73826468 & 1,01523068 & 2,723034 \\
\hline 80 & 3,34 & 47,9125 & 0,0208714 & 0,2994012 & 0,0697104 & 3,86937643 & 1,20597081 & 2,66340562 \\
\hline 100 & 3,82 & 60,1125 & 0,0166355 & 0,2617801 & 0,0635475 & 4,09621781 & 1,34025042 & 2,75596738 \\
\hline 120 & 4,96 & 71,9 & 0,0139082 & 0,2016129 & 0,0689847 & 4,27527626 & 1,60140574 & 2,67387052 \\
\hline
\end{tabular}

Isotherm Chromium adsorption repport : CA-HT

\begin{tabular}{|r|r|r|c|r|c|r|r|c|}
\hline 0 & 0 & 0 & \#DIV/0! & \#DIV/0! & \#DIV/0! & \#NOMBRE! & \#NOMBRE! & \#DIV/0! \\
\hline 40 & 0,87 & 24,45625 & 0,0408893 & 1,1494253 & 0,0355737 & 3,19688581 & $-0,1392621$ & 3,33614787 \\
\hline 60 & 1,01 & 36,86875 & 0,0271232 & 0,990099 & 0,0273945 & 3,60736431 & 0,00995033 & 3,59741398 \\
\hline 70 & 1,17 & 43,01875 & 0,0232457 & 0,8547009 & 0,0271974 & 3,76163607 & 0,15700375 & 3,60463232 \\
\hline 80 & 1,39 & 49,13125 & 0,0203536 & 0,7194245 & 0,0282916 & 3,89449529 & 0,32930375 & 3,56519154 \\
\hline 100 & 1,96 & 61,275 & 0,0163199 & 0,5102041 & 0,0319869 & 4,11537193 & 0,67294447 & 3,44242746 \\
\hline 120 & 8,15 & 69,90625 & 0,0143049 & 0,1226994 & 0,1165847 & 4,24715506 & 2,09801793 & 2,14913713 \\
\hline
\end{tabular}

Isotherm Chromium adsorption repport : CA-BA

Thermodynamic chromium adsorption repport : CA-BA

\begin{tabular}{|c|c|c|c|c|c|c|c|}
\hline $\mathrm{Ci}$ & $\mathrm{Ce}$ & $\mathrm{Q}_{\mathrm{eq}}$ & $\mathrm{K}_{\mathrm{d}}$ & $\operatorname{lnk}_{d}$ & $\mathrm{~T} \mathrm{C}$ & T K & $1 / \mathrm{T}$ \\
\hline 60 & 9,99906 & 100,00188 & 10,0011281 & 2,3026979 & 40 & 313 & 0,0031949 \\
\hline 60 & 6,93405 & 106,1319 & 15,3059035 & 2,7282386 & 60 & 333 & 0,003003 \\
\hline 60 & 4,27182 & 111,45636 & 26,0910713 & 3,26159316 & 80 & 353 & 0,0028329 \\
\hline 60 & 2,10876 & 115,78248 & 54,90548 & 4,00561316 & 100 & 373 & 0,002681 \\
\hline 60 & 4,93738 & 110,12524 & 22,3043882 & 3,10478344 & 120 & 393 & 0,0025445 \\
\hline \multicolumn{8}{|c|}{ Thermodynamic chromium adsorption report : CA-HT } \\
\hline & & 0 & \#DIV/0! & \#DIV/0! & 40 & 313 & 0,0031949 \\
\hline 60 & 8,59795 & 102,8041 & 11,9568153 & 2,48130143 & 60 & 333 & 0,003003 \\
\hline 60 & 7,93239 & 104,13522 & 13,1278492 & 2,57473587 & 80 & 353 & 0,0028329 \\
\hline 60 & 5,27016 & 109,45968 & 20,7697072 & 3,03349554 & 100 & 373 & 0,002681 \\
\hline 60 & 4,27016 & 111,45968 & 26,1019915 & 3,26201161 & 120 & 393 & 0,0025445 \\
\hline
\end{tabular}

